\title{
Dynamics of a Non-Autonomous ODE System Occurring in Coagulation Theory
}

\author{
F.P. da Costa $^{1,2,3}$ and Rafael Sasportes ${ }^{1}$
}

Received March 22, 2006

\begin{abstract}
We consider a constant coefficient coagulation equation with Becker-Döring type interactions and power law input of monomers $J_{1}(t)=\alpha t^{\omega}$, with $\alpha>$ 0 and $\omega>-\frac{1}{2}$. For this infinite dimensional system we prove solutions converge to similarity profiles as $t$ and $j$ converge to infinity in a similarity way, namely with either $j / \varsigma$ or $(j-\varsigma) / \sqrt{\varsigma}$ constants, where $\varsigma=\varsigma(t)$ is a function of $t$ only. This work generalizes to the non-autonomous case a recent result of da Costa et al. (2004). Markov Processes Relat. Fields 12, 367-398. and provides a rigorous derivation of formal results obtained by Wattis J. Phys. A: Math. Gen. 37, 7823-7841. The main part of the approach is the analysis of a bidimensional non-autonomous system obtained through an appropriate change of variables; this is achieved by the use of differential inequalities and qualitative theory methods. The results about rate of convergence of solutions of the bidimensional system thus obtained are fed into an integral formula representation for the solutions of the infinite dimensional system which is then estimated by an adaptation of methods used by da Costa et al. (2004). Markov Processes Relat. Fields 12, 367-398.
\end{abstract}

KEY WORDS: dynamics of non-autonomous ODEs; coagulation equations; self-similar behaviour; asymptotic evaluation of integrals.

AMS SUBJECT CLASSIFICATION NUMBERS: 34C05; 34C11; 34C20; 34D99; 82C21.

\section{INTRODUCTION}

The dynamic behaviour of differential equations modelling the kinetics of cluster growth has received a great deal of attention in the last decade or so (for a recent review see [8]).

\footnotetext{
${ }^{1}$ Universidade Aberta, DCET, Rua Fernão Lopes 9, 2oDto, P-1000-132 Lisboa, Portugal.

${ }^{2}$ Instituto Superior Técnico, CAMGSD, Av. Rovisco Pais 1, 1049-001 Lisboa, Portugal.

E-mail: Fcosta@univ-ab.pt

${ }^{3}$ To whom correspondence should be addressed.
} 
One of the questions currently commanding widespread attention is whether or not similarity behaviour occurs in these systems. Roughly speaking, similarity (or self-similar, or self-preserving) behaviour means the existence of a universal function to which all solutions converge at large times after an appropriate scaling transformation (see below). This is believed to take place for large classes of cluster systems and, although a general solution is still lacking, considerable progress has recently been made (see, e.g. [6,9-12]). In the present paper, we shall address this question for a particular type of cluster system, namely a Smoluchowski equation with Becker-Döring type interactions (also called addition model in the literature $[4,7])$ with time dependent input of monomers. In this Introduction, we start by presenting the system and the problem to be dealt with, and then we give a brief description of the approach used and the results contained in the rest of the paper.

Consider the following coagulation system: suppose we have a system of clusters with masses $j \in \mathbb{N}^{+}$and we are interested in a mean field description where the only relevant quantities are the time dependent concentration of each cluster of mass $j$ ( $j$-cluster for short, a 1-cluster is called a monomer). Assuming the system is sufficiently diluted so that the only relevant interactions are those between clusters and monomers (and no interactions of cluster-cluster type occur), and the evolution of the cluster population can be described by the mass action law, we have the following kinetic scheme

$$
(j \text {-cluster })+(1 \text {-cluster }) \stackrel{a_{j, 1}}{\longrightarrow}((j+1) \text {-cluster }), \quad j \in \mathbb{N}^{+},
$$

where $a_{j, 1}$ is the kinetic constant for the reaction; to this scheme corresponds the following coagulation kinetic equations

$$
\begin{aligned}
& \dot{c}_{1}=-a_{1} c_{1}^{2}-c_{1} \sum_{j=1}^{\infty} a_{j} c_{j}, \\
& \dot{c}_{j}=a_{j-1} c_{1} c_{j-1}-a_{j} c_{1} c_{j}, \quad j \geqslant 2,
\end{aligned}
$$

with $c_{j}=c_{j}(t)$ denoting the concentration of the $j$-cluster at time $t$, and the coefficients are $a_{1}=\frac{1}{2} a_{1,1}$ and $a_{j}=a_{j, 1}$ if $j \geqslant 2$.

Due to the special role played by the monomers in the kinetic scheme, we expect the dynamics of the differential equation to freeze when the system runs out of monomers, and the final state will clearly depend on the initial distribution of clusters sizes. The problem only becomes interesting if some mechanism is introduced that avoids the complete depletion of monomers. One such case, widely considered in the literature, is the possibility of clusters to fragment (i.e., the kinetic scheme to be 
reversible) which gives rise to the classical dynamic Becker-Döring system [2]; another possibility is to externally provide a source of monomers by adding a source term $J_{1}(t)$ to the right hand side of the $c_{1}$-equation in (1). The particular type of input function to be considered clearly depends on the particular situation to be modelled. Several possibilities have been considered in the literature. Among them, we can point the case where $J_{1}(t)$ exactly balances the remaining terms in the right hand side of the $c_{1}$-equation, in which case that differential equation reduces to $\dot{c}_{1}=0$; this case corresponds, physically, to the coupling of the cluster system with an infinite particle bath of monomers that allows their concentration to be constant in time, and, in fact, constitutes the original Becker-Döring theory of metastability [13].

Another way to externally supply monomers is to define the input term $J_{1}(t)$ independently of the state of the system. This is a quite reasonable assumption in a number of applications, including in simple models of polymerization and of epitaxial growth [3]. The easiest hypothesis about $J_{1}(t)$, which turns out to be very useful in applications, is to make it a time independent constant. This case was considered recently in [5] (see below). Another model case for the input function is to consider it a power law $J_{1}(t)=\alpha t^{\omega}$, with $\alpha>0$ and $\omega \in \mathbb{R}$ constants. This case is possibly not as interesting for the applications as the time independent case $(\omega=0)$, but it is still a case deserving consideration, and constitutes an interesting mathematical challenge, since the approach used in [5] is based on a compactification method and centre manifold reduction techniques, that clearly are not available for the power law case. A formal analysis of this case was recently performed in [14], and in this paper we use the ansatz provided by that study to rigorously analyse the addition model with constant rate coefficients $a_{j} \equiv 1$ and a power law input of monomers $J_{1}(t)=\alpha t^{\omega}$, namely

$$
\begin{aligned}
& \dot{c}_{1}=\alpha t^{\omega}-c_{1}^{2}-c_{1} \sum_{j=1}^{\infty} c_{j}, \\
& \dot{c}_{j}=c_{1} c_{j-1}-c_{1} c_{j}, \quad j \geqslant 2,
\end{aligned}
$$

where $\alpha>0$ and $\omega>-\frac{1}{2}$ are constant parameters. The case $\omega \leqslant-\frac{1}{2}$, which based on [14] is expected to give a different result, will be the subject of a later work.

There are basically two aspects of the dynamical behaviour of solutions to (2) that concern us in this paper.

First, we want to establish the componentwise behaviour of the solution as $t \rightarrow+\infty$, as well as the behaviour of the total amount of clusters present (the bulk), defined by $\sum_{j=1}^{\infty} c_{j}$. This will be stated in 
Theorem 2 and Section 3, which roughly says that, as $t \rightarrow+\infty, c_{j}(t) \sim$ $t^{\frac{\omega-1}{3}}, \sum_{j=1}^{\infty} c_{j}(t) \sim t^{\frac{2 \omega+1}{3}}$ and $\alpha t^{\omega}-c_{1}(t) \sum_{j=1}^{\infty} c_{j}(t) \sim t^{2 \frac{\omega-1}{3}}$.

The second aspect of the dynamics we are interested in is the occurrence of similarity behaviour. As pointed out above, this means that the cluster size distribution approach a common profile at large times, more precisely, there exist functions $\Phi$ and $\zeta$, and positive constants $a$ and $b$, such that, for sufficiently large $j$ and $t$,

$$
c_{j}(t) \sim \varsigma(t)^{-a} \Phi\left(j \varsigma(t)^{-b}\right) .
$$

For a thorough discussion of similarity behaviour in coagulating systems (see [9]). Here we will centre our attention in the mathematical aspects of such behaviour for system (2). The relevant results are stated and proved in Theorem 3 and Section 4, and basically establish the existence of a function $\zeta(t) \sim t^{\frac{\omega+2}{3}}$ and a family of scaling functions $\Phi_{1, \omega}$ such that (3) holds with $a=\frac{1-\omega}{2+\omega}$ and $b=1$. The graphs of $\eta \mapsto \Phi_{1, \omega}(\eta)$ are presented in Figure 7. The case $\omega=0$ was already known from [5] and is now seen to be part of a more general picture. Our results do not quite match the formal results presented in [14] since the scaling transformation $t \mapsto \varsigma(t)$ is not the same in both cases. Considering that all our $\Phi_{1, \omega}$ have a singular point at precisely $\eta=1$, independently of $\omega$ (although the type of singularity does depend on $\omega$, see Figure 7), whereas the singular point of the corresponding functions in [14] vary with $\omega$, we think out result presents a simpler description of the similarity behaviour.

From the observation that $\Phi_{1, \omega}$ becomes discontinuous at $\eta=1$ for $\omega \leqslant 1$, and unbounded in any set $(1-\varepsilon, 1)$ when $\omega<1$, it is reasonable to infer that the scaling under consideration may not be the appropriate one around that point. A different scaling variable to deal with this case was successfully developed in [5] for the autonomous case $\omega=0$. It was also applied in [14] to the present case where a different scaling function was deduced. The remainder of Section 4, in particular Theorem 4, states and rigorously proves this different scaling behaviour, and in the process greatly simplifies the original writing of the scaling function in [14], Eq. (3.29), which resorted to Kummer's hypergeometric functions, by showing it to be the rather pleasent looking function $\Phi_{2, \omega}$ in (45), that reduces to the one obtained in [5] when $\omega=0$. Then, we essentially have, for sufficiently large $j$ and $t$,

$$
c_{j}(t) \sim \varsigma(t)^{-a / 2} \Phi_{2, \omega}\left(\frac{j-\varsigma(t)}{\sqrt{\varsigma(t)}}\right)
$$

with $a$ and $\varsigma(t)$ as before. The graphs of the new scaling functions $\Phi_{2, \omega}$ are presented in Figure 8. Note that they provide a kind of inner 
expansion of the singularity of the scaling functions $\Phi_{1, \omega}$ at $\eta=1$. Again in this case, the situation for $\omega=0$ had already been proved, and is now shown to be part of a more general setting.

The proofs of the just described results of Sections 3 and 4 are achieved due to essentially two arguments that help transforming the infinite dimensional system (2) into an almost exactly solvable problem. The first of them is the fact that by introducing the total number of clusters as a new macroscopic variable $c_{0}(t)$ defined by

$$
c_{0}(t)=\sum_{j=1}^{\infty} c_{j}(t)
$$

and formally differentiating termwise, we conclude that $c_{0}$ satisfies the evolution equation $\dot{c}_{0}=\alpha t^{\omega}-c_{0} c_{1}$. Thus, system (2) can, at least formally, be written, in closed form, as

$$
\begin{aligned}
& \dot{c}_{0}=\alpha t^{\omega}-c_{0} c_{1}, \\
& \dot{c}_{1}=\alpha t^{\omega}-c_{0} c_{1}-c_{1}^{2}, \\
& \dot{c}_{j}=c_{1} c_{j-1}-c_{1} c_{j}, \quad j \geqslant 2 .
\end{aligned}
$$

In fact, the equivalence between the dynamics of (2) and that of (5) with initial data in the hyperplane $\left\{\left(c_{0}, c_{1}, \ldots\right) \in \mathbb{R}^{\mathbb{N}}: c_{0}-\sum_{j=1}^{\infty} c_{j}=0\right\}$ can be proved as in Section 2 of Ref. [5]. From the reduced system (5) we observe that the equations governing both the monomer dynamics and the total number of clusters are actually a nonautonomous bidimensional $\left(c_{0}, c_{1}\right)$ system

$$
\begin{aligned}
& \dot{c}_{0}=\alpha t^{\omega}-c_{0} c_{1}, \\
& \dot{c}_{1}=\alpha t^{\omega}-c_{0} c_{1}-c_{1}^{2}
\end{aligned}
$$

the dynamics of which can be studied in a way totally independent of the remaining components of the infinite dimensional system (more about this later).

The second argument alluded to above has to do with a change of time scale. Given a solution of (6), introduce a new time scale

$$
\varsigma(t):=\varsigma_{0}+\int_{t_{0}}^{t} c_{1}(s) d s,
$$

where $\varsigma_{0}$ is a positive constant, and consider the new phase variables

$$
\widetilde{c}_{j}(\varsigma):=c_{j}(t(\varsigma))
$$


where $t(\varsigma)$ is the inverse function of $\varsigma(t)$. When $c_{1}(t)>0$, these are well defined and $\varsigma$ is an increasing function of $t$. In the new variables, the $c_{j}$-equations in (5) become

$$
\tilde{c}_{j}{ }^{\prime}=\tilde{c}_{j-1}-\tilde{c}_{j}, \quad j \geqslant 2,
$$

where $(\cdot)^{\prime}=\frac{d}{d s}$. This system of differential equations is a lower triangular linear system and thus can be explicitly solved in terms of the function $\widetilde{c}_{1}(\varsigma)$ starting from the equation for $j=2$ and applying the variation of constants formula recursively:

$$
\tilde{c}_{j}(\varsigma)=e^{-\varsigma} \sum_{k=2}^{j} \frac{\varsigma^{j-k}}{(j-k) !} c_{k}(0)+\frac{1}{(j-2) !} \int_{0}^{\varsigma} \widetilde{c}_{1}(\varsigma-s) s^{j-2} e^{-s} d s .
$$

Once a precise knowledge of the behaviour of $\widetilde{c}_{1}$ is available, this representation formula for the $j$-component of the solution in terms of $\widetilde{c}_{1}(\varsigma)$ allows us to prove the rather detailed information about the long time evolution of solutions to (2) that is presented in Sections 3 and 4. The methods used in the analysis of the representation formula (9) follow closely those developed for the corresponding results in [5] and so, in those two Sections, we merely describe the main steps of the arguments, directing the reader to da Costa et al. [5] for full details. What is completely new in this paper is the way the relevant information about $\widetilde{c}_{1}$ is now obtained. That information is stated in Theorem 2, the proof of which takes the full content of Section 2. In the remaining of this Introduction we shall briefly describe the main ideas involved.

What we need to know about the behaviour of $\widetilde{c}_{1}(\varsigma)$ is not only its limit as $\varsigma \rightarrow+\infty$ but the actual rate of convergence. For the autonomous $\omega=0$ case studied in [5] this information is obtained from the study of (6) using invariant regions, a change of phase and independent variables suggested by Poincarés compactification, and a centre manifold analysis. This approach is not available for the general $\omega \neq 0$ case, and so, here, we use an indirect path, which starts by making an ansatz for a convenient change of variables, suggested by Wattis [14], Table 2 and obtained via formal asymptotics.

According to Wattis [14] we expect solutions $\left(c_{0}, c_{1}\right)$ of system (6) with $\alpha>0$ and $\omega>-\frac{1}{2}$ to behave like

$$
\begin{aligned}
& c_{0}(t) \sim\left(\frac{3 \alpha^{2}}{1+2 \omega}\right)^{\frac{1}{3}} t^{\frac{1+2 \omega}{3}}, \\
& c_{1}(t) \sim\left(\frac{\alpha(1+2 \omega)}{3}\right)^{\frac{1}{3}} t^{\frac{\omega-1}{3}},
\end{aligned}
$$


as $t \rightarrow+\infty$. This suggests the functions

$$
\begin{aligned}
& C_{0}(t):=\left(\frac{1+2 \omega}{3 \alpha^{2}}\right)^{\frac{1}{3}} t^{-\frac{1+2 \omega}{3}} c_{0}(t) \\
& C_{1}(t):=\left(\frac{3}{\alpha(1+2 \omega)}\right)^{\frac{1}{3}} t^{\frac{1-\omega}{3}} c_{1}(t)
\end{aligned}
$$

might be expected to converge to 1 as $t \rightarrow+\infty$, and reciprocally, if this happens then $c_{0}$ and $c_{1}$ behave as stated. To prove this convergence behaviour of $\left(C_{0}, C_{1}\right)$ we need an equation for its evolution which is readily obtained as follows: differentiating (10) and (11), substituting into (6), changing the time scale $t \mapsto \tau$ so that $\frac{d \tau}{d t}=\left(\frac{3 \alpha^{2}}{1+2 \omega}\right)^{\frac{1}{3}} t^{\frac{1+2 \omega}{3}}$, defining

$$
\begin{aligned}
& x(\tau):=C_{1}(t(\tau)), \\
& y(\tau):=C_{0}(t(\tau))
\end{aligned}
$$

and denoting $\frac{d}{d \tau}(\cdot)$ by $(\cdot)^{\prime}$, we obtain

$$
\begin{aligned}
& x^{\prime}=1-x y-A \tau^{-\frac{1}{2}} x^{2}+B \tau^{-1} x, \\
& y^{\prime}=(1-x y) A \tau^{-\frac{1}{2}}-A^{2} \tau^{-1} y,
\end{aligned}
$$

where

$$
\begin{aligned}
A & :=\left(\frac{1+2 \omega}{4+2 \omega}\right)^{\frac{1}{2}}, \\
B & :=\frac{1-\omega}{4+2 \omega} .
\end{aligned}
$$

To prove that non-negative solutions to (14) converge to $(1,1)$ as $\tau \rightarrow$ $+\infty$ is the objective of Section 2. To this end we proceed as follows: first we prove, in Lemma 1 , that $\mathbb{R}^{+} \times \mathbb{R}^{+}$is a positively invariant set for (14); then a relative boundedness result is obtained: we establish in Lemma 2 that, for non-negative solutions $(x, y)$, boundedness of $x$ [or $y$ ] is equivalent to boundedness away from zero of $y$ [or $x$ ]; and then, in Lemma 3, we conclude that indeed both $x$ and $y$ are bounded (and bounded away from zero) functions of $\tau$. The proofs of all these three lemmas are achieved by the use of appropriate differential inequalities.

After these boundedness results we use the evolution of two auxiliary functions, $h(\tau):=x(\tau) y(\tau)$ and $b(\tau)=y(\tau)-A \tau^{-\frac{1}{2}} x(\tau)$, to locate, for every orbit, its $\omega$-limit set as a (bounded) arc of the hyperbola $x y=1$ containing the point $(1,1)$ (Lemmas 4 and 5) and, using suitable differential inequalities akin to those employed in the boundedness proof (Lemma 3), 
we finally establish that the arc actually degenerates into the single point $(1,1)$ (Lemma 6).

Using this convergence it is possible to refine the estimates for $h$ presented in Lemma 4 and actually conclude that, as $\tau \rightarrow+\infty, h(\tau) \sim 1-$ $A \tau^{-\frac{1}{2}}$. This estimate, obtained in Lemma 7, concludes the information required for the proofs of the results on the dynamics of (2) in Sections 3 and 4 we described above.

The approach just outlined, when compared with the more natural one used in the autonomous $\omega=0$ case, has the great disadvantage of, besides being lengthier, requiring an a priori knowledge about the behaviour to be observed in order to be able to define the appropriate change of variables. This intrinsic drawback is in this case mitigated by our knowledge of the stated behaviour from the formally computed asymptotic results presented in [14], Table 2.

\section{THE BIDIMENSIONAL NON-AUTONOMOUS ODE GOVERNING MONOMER AND BULK DYNAMICS}

We are interested in non-negative solutions to (6) and so, from hereon, solutions will actually mean non-negative solutions. The main result of this section is the following

Theorem 1. Let $\alpha>0$ and $\omega>-\frac{1}{2}$ be constants, and let $\left(c_{0}, c_{1}\right)$ be any solution of (6). Then, as $t \rightarrow+\infty$, we have

$$
\begin{aligned}
\text { (i) } & \left(\frac{1+2 \omega}{3 \alpha^{2}}\right)^{\frac{1}{3}} t^{-\frac{1+2 \omega}{3}} c_{0}(t) \longrightarrow 1, \\
\text { (ii) } & \left(\frac{3}{\alpha(1+2 \omega)}\right)^{\frac{1}{3}} t^{\frac{1-\omega}{3}} c_{1}(t) \longrightarrow 1, \\
\text { (iii) } & \left(\frac{3}{\alpha(1+2 \omega)}\right)^{\frac{2}{3}} t^{2 \frac{1-\omega}{3}}\left(\alpha t^{\omega}-c_{0}(t) c_{1}(t)\right) \longrightarrow 1 .
\end{aligned}
$$

From the discussion in the Introduction we prove this result by working with (14) and proving that its solutions converge to $(1,1)$ as $\tau \rightarrow+\infty$.

We start by the obvious observation that (14) is asymptotically autonomous. Although in general this would be a potentially fruitful property, the fact that the set of equilibria of the asymptotic limit system

$$
\begin{aligned}
& x^{\prime}=1-x y, \\
& y^{\prime}=0
\end{aligned}
$$

form a continuum, and thus the equilibria are non-hyperbolic, does not seem to provide a handy way of using the limit system to get information 
about the long time behaviour of solutions to the original non-autonomous system (14).

As a consequence, we shall not use the asymptotic limit of (14) in this paper. Instead, as described in the Introduction, we study (14) using the information on the boundedness of solutions provided by certain differential inequalities derived from (14) in some regions of the phase plane $(x, y)$, together with information provided by the evolution of two auxiliary functions, to locate the possible $\omega$-limit set of the solutions, and then to prove this set must consist of the single point $(1,1)$. The somewhat lengthy analysis will be decoupled into a series of lemmas in a way that will highlight the main steps of the argument.

Our first goal is to establish that all (positive) solutions to (14) have both components bounded and bounded away from zero. To this end, we start by proving solutions cannot cease being positive.

Lemma 1. The first quadrant $\{x \geqslant 0, y \geqslant 0\}$ is positively invariant for (14).

Proof. This is obvious since in $\{(0, y): y \geqslant 0\}$ we have, from (14), $x^{\prime}=$ $1>0$ for all $\tau$, and so solutions cannot escape through the $y$-axis. Similarly, in $\{(x, 0): x \geqslant 0\}$ it holds $y^{\prime}=A \tau^{-\frac{1}{2}}>0$, which imply solutions cannot escape through the $x$-axis either.

Next we prove a relative boundedness behaviour of the $x$ and $y$ components of the solution that will be useful in the sequel.

Lemma 2. Let $(x, y)$ be any solution to (14) with positive initial data. Then, the following equivalences hold:

(i) $y$ is bounded $\Longleftrightarrow x$ is bounded away from zero.

(ii) $y$ is bounded away from zero $\Longleftrightarrow x$ is bounded.

Proof. We start by proving that

$y$ is bounded $\Longrightarrow x$ is bounded away from zero.

When $\tau \in\left(\tau_{0}, 1\right]$ (if this set is non empty) the solution $x(\tau)$ is necessarily bounded away from zero since otherwise there would exist at least a point $\tilde{\tau} \in\left(\tau_{0}, 1\right]$ for which $x(\widetilde{\tau})=0$, and thus $x^{\prime}(\widetilde{\tau})=1>0$, which is impossible because then $x(\tau)<0$, for $\tau<\tilde{\tau}$ sufficiently close to $\tilde{\tau}$, contradicting Lemma 1. When $\tau>1$, let $U_{y}>0$ be an upper bound for $y$. For the case $\omega \geqslant 1$, for which $B \leqslant 0$, the first equation in (14) gives $x^{\prime} \geqslant 1-x U_{y}-$ $A \tau^{-\frac{1}{2}} x^{2}-|B| \tau^{-1} x>1-\left(U_{y}+|B|\right) x-A x^{2}$. In the case $\omega \in\left(-\frac{1}{2}, 1\right)$, we 
have $B>0$, and hence (14) results in $x^{\prime} \geqslant 1-x U_{y}-A \tau^{-\frac{1}{2}} x^{2}+B \tau^{-1} x>$ $1-x U_{y}-A x^{2}$. In either case, standard results on differential inequalities imply $x(\tau)$ is bounded away from zero.

To prove the reverse result,

$x$ is bounded away from zero $\Longrightarrow y$ is bounded,

we notice that the equation for $y$ in (14) is linear in $y$. In fact it can be written as

$$
y^{\prime}=-\left(x+A \tau^{-\frac{1}{2}}\right) A \tau^{-\frac{1}{2}} y+A \tau^{-\frac{1}{2}} .
$$

Changing to a new time scale $\tau \mapsto \theta$ with $\frac{d \theta}{d \tau}=A \tau^{-\frac{1}{2}}$, the equation for $y$ becomes

$$
\frac{d y}{d \theta}=-\left(x+\frac{2 A^{2}}{\theta}\right) y+1
$$

Since this a linear equation with differentiable coefficients, it is obvious the solution is bounded for $\theta$ in every bounded set, so the problem reduces to the study of $y(\theta)$ when $\theta \rightarrow+\infty$. By the variation of constants formula we get

$$
y(\theta)=y\left(\theta_{0}\right) e^{-\int_{\theta_{0}}^{\theta}\left(x(\sigma)+\frac{2 A^{2}}{\sigma}\right) d \sigma}+\int_{\theta_{0}}^{\theta} e^{-\int_{s}^{\theta}\left(x(\sigma)+\frac{2 A^{2}}{\sigma}\right) d \sigma} d s .
$$

Now let $L_{x}>0$ be a lower bound for $x$. Then, we have

$$
e^{-\int_{\theta_{0}}^{\theta}\left(x(\sigma)+\frac{2 A^{2}}{\sigma}\right) d \sigma} \leqslant e^{-L_{x}\left(\theta-\theta_{0}\right)} e^{2 A^{2} \log \left(\theta_{0} / \theta\right)}=\left(\frac{\theta_{0}}{\theta}\right)^{2 A^{2}} e^{-L_{x}\left(\theta-\theta_{0}\right)} \longrightarrow 0
$$

as $\theta \rightarrow+\infty$. Also,

$$
\int_{\theta_{0}}^{\theta} e^{-\int_{s}^{\theta}\left(x(\sigma)+\frac{2 A^{2}}{\sigma}\right) d \sigma} d s \leqslant \int_{\theta_{0}}^{\theta} e^{-L_{x}(\theta-s)} d s=\frac{1-e^{-L_{x}\left(\theta-\theta_{0}\right)}}{L_{x}}<\frac{1}{L_{x}},
$$

and plugging these into (17) we conclude the boundedness of $y$, concluding the proof of part (i).

Let us now consider the case

$$
y \text { is bounded away from zero } \Longrightarrow x \text { is bounded. }
$$

Let $L_{y}>0$ be a positive lower bound for $y$. Then, $x^{\prime} \leqslant 1-x L_{y}-A \tau^{-\frac{1}{2}} x^{2}+$ $B \tau^{-1} x<1-\left(L_{y}-B \tau^{-1}\right) x$. When $\omega \geqslant 1$ we have $B \leqslant 0$ and thus $x^{\prime}<$ 
$1-L_{y} x$ and by standard differential inequalities results we conclude the boundedness of $x$ :

$x(\tau) \leqslant x\left(\tau_{0}\right) e^{-L_{y}\left(\tau-\tau_{0}\right)}+\int_{\tau_{0}}^{\tau} e^{-L_{y}(\tau-s)} d s=\mathcal{O}(1)+\frac{1-e^{-L_{y}\left(\tau-\tau_{0}\right)}}{L_{y}}<\mathcal{O}(1)+\frac{1}{L_{y}}$

as $\tau \rightarrow+\infty$. When $\omega \in\left(-\frac{1}{2}, 1\right)$ we have $B>0$. For this case, let $\tilde{\tau}=2 B / L_{y}$. For $\tau>\tilde{\tau}$ it follows that $L_{y}-B \tau^{-1}>L_{y}-B \widetilde{\tau}^{-1}=L_{y} / 2$, and thus $x^{\prime}<$ $1-\frac{L_{y}}{2} x$. Now, for $\tau \in\left(\tau_{0}, \widetilde{\tau}\right)$, we have $x(\tau) \leqslant u(\tau)$ where $u$ is the solution of $u^{\prime}=1-\left(L_{y}-B \tau^{-1}\right) u$ with $u\left(\tau_{0}\right)=x\left(\tau_{0}\right)$, which is obviously a bounded function in the compact interval $\left[\tau_{0}, \tilde{\tau}\right]$. For $\tau \geqslant \tilde{\tau}$ we just repeat the argument employed in the case $\omega \geqslant 1$ with $L_{y}$ changed to $L_{y} / 2$.

Finally, it remains to be proved that

$x$ is bounded $\Longrightarrow y$ is bounded away from zero.

Let $U_{x}$ be an upper bound for $x$. By (17), the variation of constants formula for $y$, we can write

$$
\begin{aligned}
y(\theta) & \geqslant \int_{\theta_{0}}^{\theta} e^{-U_{x}(\theta-s)} e^{-\int_{s}^{\theta} \frac{2 A^{2}}{\sigma} d \sigma} d s \\
& \geqslant \int_{\theta_{0}}^{\theta} e^{-\left(U_{x}+\frac{2 A^{2}}{\theta_{0}}\right)(\theta-s)} d s \\
& =\frac{1}{U_{x}+\frac{2 A^{2}}{\theta_{0}}}\left(1-e^{-\left(U_{x}+\frac{2 A^{2}}{\theta_{0}}\right)\left(\theta-\theta_{0}\right)}\right) \underset{\theta \rightarrow+\infty}{\longrightarrow} \frac{1}{U_{x}+\frac{2 A^{2}}{\theta_{0}}}>0 .
\end{aligned}
$$

Hence, for all $\theta$ sufficiently large, we certainly have $y$ bounded away from zero, and for $\theta$ in compact intervals $y$ is clearly bounded away from zero being the sum of two differentiable positive functions.

This concludes the proof of Lemma 2 .

The equivalences of Lemma 2 roughly imply that the only possibilities left for solutions to escape to infinity are along the coordinate axis. In the next lemma we prove this does not occur, and solutions are, in fact, bounded.

Lemma 3. Every solution $(x, y)$ to (14) with positive initial data is bounded.

Proof. We separate the proof into two steps, studying first the boundedness of $y$, and then that of $x$.

Step 1: the boundedness of $y(\tau)$. 


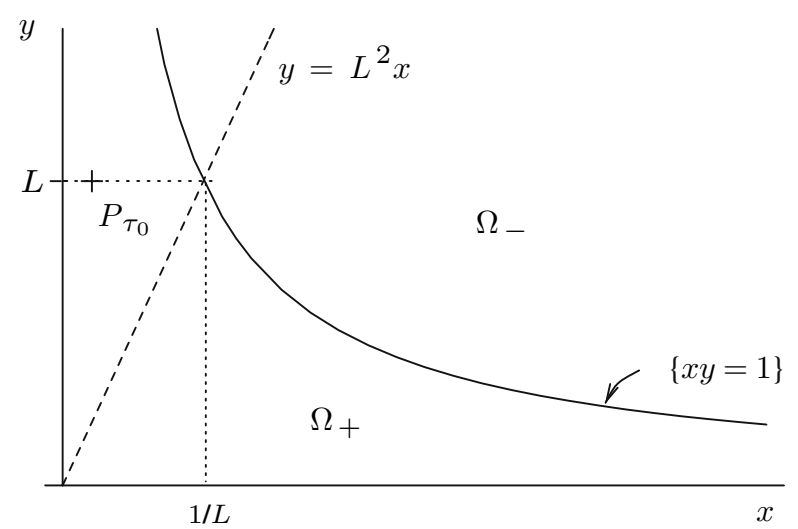

Figure 1. Situation described in the text for a point of an orbit having $y(\tau) \rightarrow+\infty$ as $\tau \rightarrow+\infty$.

It is easy to conclude that the only possibility for the orbit to have an unbounded $y$ component is that it leaves the region $\Omega_{-}:=\{(x, y): x y \geqslant 1\}$. In fact, for $x y \geqslant 1$ we have, from the second equation in (14),

$$
y^{\prime}=\left((1-x y)-A \tau^{-\frac{1}{2}} y\right) A \tau^{-\frac{1}{2}} \leqslant-A^{2} \tau^{-1} y<0
$$

and so $y(\tau)$ cannot diverge to $+\infty$ while staying in $\Omega_{-}$. We now prove that it cannot diverge to $+\infty$ when the orbit is in $\Omega_{+}:=\{(x, y): 0<x y<1\}$ either, and so the orbit must have a bounded $y$ component. Let $L>1$ be fixed arbitrarily and define $\tau_{L}:=2^{-2}\left(L^{2}-L^{-1}\right)^{-2}$. If $y(\tau)<L$ for all sufficiently large $\tau$, then $y$ is bounded and there is nothing to prove. Suppose there exists a $\tau_{0}>\max \left\{1, \tau_{L}\right\}$ such that $y\left(\tau_{0}\right)=L$ and $P_{\tau_{0}}=\left(x\left(\tau_{0}\right), y\left(\tau_{0}\right)\right) \in$ $\Omega_{+}$. Thus we have $y\left(\tau_{0}\right)>L^{2} x\left(\tau_{0}\right)$ (see Fig. 1).

Since (15) and (16) imply that $-B A^{-1}<1 / 2$, it follows that, for all $\tau \geqslant \tau_{0}$ for which the solution satisfies $x<L^{-1}$ and $y>L^{2} x$, which clearly covers the region of interest, the following inequality holds true

$$
\begin{aligned}
y^{\prime} & =\left(1-x y-A \tau^{-\frac{1}{2}} y\right) A \tau^{-\frac{1}{2}} \\
& =\left(x^{\prime}+A \tau^{-\frac{1}{2}} x^{2}-B \tau^{-1} x-A \tau^{-\frac{1}{2}} y\right) A \tau^{-\frac{1}{2}} \\
& <\left(x^{\prime}+\left(x-L^{2}-B A^{-1} \tau^{-\frac{1}{2}}\right) x A \tau^{-\frac{1}{2}}\right) A \tau^{-\frac{1}{2}} \\
& <\left(x^{\prime}+\left(L^{-1}-L^{2}+\frac{1}{2} \tau^{-\frac{1}{2}}\right) x A \tau^{-\frac{1}{2}}\right) A \tau^{-\frac{1}{2}} \\
& <x^{\prime} A \tau^{-\frac{1}{2}},
\end{aligned}
$$

where the last inequality arises from our lower bound on $\tau_{0}$. 


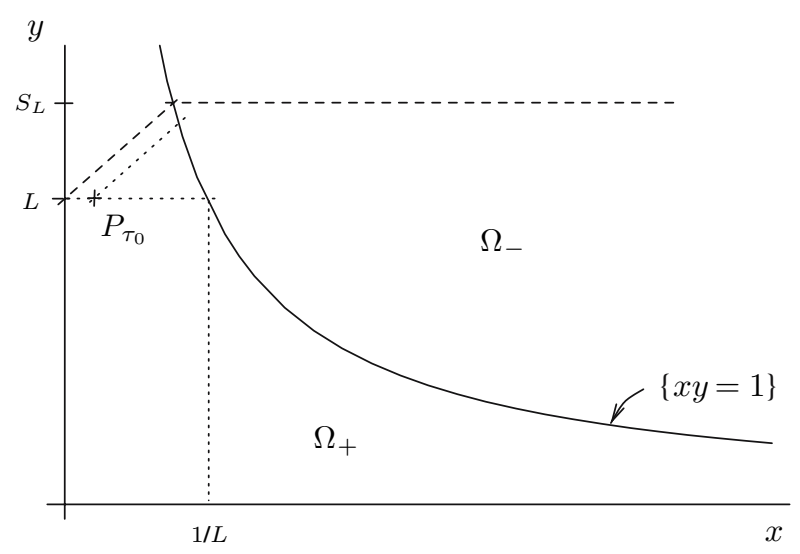

Figure 2. Bounding region for an orbit satisfying $y\left(\tau_{0}\right)=L>1$ in some point $P_{\tau_{0}} \in \Omega_{+}$. Note that $y^{\prime}<0$ in the closure of $\Omega_{-} . S_{L}$ is the ordinate of the intersection point of the straight line $y=x+L$ with the hyperbola $x y=1$.

Now, either $x^{\prime} \leqslant 0$, in which case $y^{\prime}<0$ and $y$ cannot increase, and thus it is bounded above, or $x^{\prime}>0$, in which case (18) and the inequalities $\tau \geqslant \tau_{0}>\max \left\{1, \tau_{L}\right\}$ imply that $y^{\prime}<x^{\prime}$ and thus $\frac{d y}{d x}<1$. Observe that this holds independently of $L$ and of the point $P_{\tau_{0}}$. This inequality shows that, the orbit's slope being uniformly bounded above by a positive constant, the orbit itself cannot rise too high in $\Omega_{+}$: the $y$ component of the solution is always bounded above by $S_{L}:=\frac{L+\sqrt{L^{2}+4}}{2}$ (see Fig. 2)

Step 2: boundedness of $x(\tau)$.

Suppose there exists an orbit such that $x(\tau) \rightarrow+\infty$ as $\tau \rightarrow+\infty$. First observe that, by Lemma 2(ii), we must have $y(\tau)$ not bounded away from zero for all sufficiently large $\tau$. In fact, the orbit must eventually enter $\Omega_{+}$: if the orbit stays in $\Omega_{-}$then $x(\tau) y(\tau)>1$ and thus, for all sufficiently large times,

$$
\begin{aligned}
x^{\prime} & =1-x y-A \tau^{-\frac{1}{2}} x^{2}+B \tau^{-1} x \\
& =1-x y+\left(-x+B A^{-1} \tau^{-\frac{1}{2}}\right) A \tau^{-\frac{1}{2}} x \\
& <0
\end{aligned}
$$

where the term in parentheses is negative since, by assumption, $x(\tau) \rightarrow+\infty$ when $\tau \rightarrow+\infty$. The same argument shows the orbit cannot leave $\Omega_{+}$infinitely often if $x(\tau)$ is diverging to $+\infty$ because, if so, we could take $\widetilde{\tau}$ and $x(\widetilde{\tau})$ as large as wanted, for which the orbit was entering $\Omega_{-}$coming from $\Omega_{+}$. At that point, we would have, from the argument above, $x^{\prime}(\tau)<0$ and so, in order for the orbit to enter $\Omega_{-}$we must have $y^{\prime}(\widetilde{\tau})>0$ (see Fig. 3). 


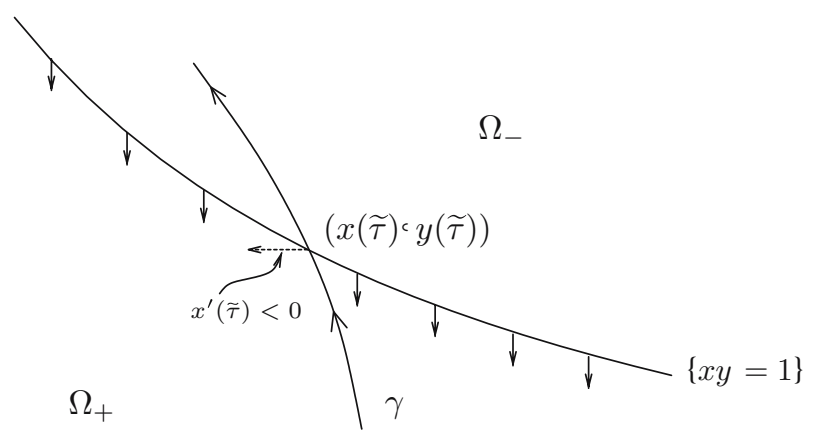

Figure 3. The impossibility of an orbit $\gamma$ to travel from $\Omega_{+}$to $\Omega_{-}$for all large enough $\tilde{\tau}$ and $x(\tilde{\tau})$.

But this is impossible since Eq. (14) implies $y^{\prime}(\widetilde{\tau})=-y(\widetilde{\tau}) A^{2} \widetilde{\tau}^{-1}<0$ for all points $(x(\widetilde{\tau}), y(\widetilde{\tau}))$ in the hyperbola $\{x y=1\}$ and $\widetilde{\tau}>0$. Consequently, the orbit for which $x(\tau)$ diverges to $+\infty$ must remain in $\Omega_{+}$after some sufficiently large time and thus $y(\tau) \rightarrow 0$ as $\tau \rightarrow+\infty$. Now, for $\tau>0$, define $g_{\tau}(x, y):=x+A \tau^{-\frac{1}{2}}-\frac{1}{y}$, and consider the curves in $\Omega_{+}$defined by the level sets $\left\{g_{\tau}=0\right\}$. These curves form a sequence of hyperbolas monotonically converging to the hyperbola $\{x y=1\}$ as $\tau \rightarrow+\infty$, uniformly in compact subsets of $\mathbb{R}^{2+}$. From (14) we deduce that, for all sufficiently large $\tau$,

$$
A \tau^{-\frac{1}{2}} x^{\prime}-y^{\prime}=-\left(x-\frac{y}{x}-\frac{B}{A} \tau^{-\frac{1}{2}}\right) A^{2} \tau^{-1} x<0 .
$$

This obviously entails that, for all $\tau$ large enough,

$$
y^{\prime}>x^{\prime} A \tau^{-\frac{1}{2}} \text {. }
$$

Hence, for all sufficiently large $\tau$, if $x^{\prime}>0$ then also $y^{\prime}>0$, and so it is clear from the geometry of the curves $g_{\tau}=0$ that the orbit under consideration must cross every one of these curves from below, i.e., from $\left\{g_{\tau}(x, y)<0\right\}$ into $\left\{g_{\tau}(x, y)>0\right\}$, or, equivalently, $g_{\tau}$ must increase along the orbit. Now, from (14) and the definition of $g_{\tau}$, we deduce

$$
g_{\tau}=0 \Rightarrow A \tau^{-\frac{1}{2}} y g_{\tau}(x, y)=0 \Leftrightarrow y^{\prime}=0 \Rightarrow x^{\prime}=A \tau^{-\frac{1}{2}}\left(y-x^{2}+\frac{B}{A} \tau^{-\frac{1}{2}} x\right),
$$

furthermore $\frac{d g_{\tau}}{d \tau}=x^{\prime}-\frac{1}{2} A \tau^{-\frac{3}{2}}+\frac{y^{\prime}}{y^{2}}$, and from these expressions we can conclude that, if $g_{\tau}=0$ then $\frac{d g_{\tau}}{d \tau}=x^{\prime}-\frac{1}{2} A \tau^{-\frac{3}{2}}<0$, which obviously contradicts the result about the crossing of $g_{\tau}=0$ from below that was obtained before. 
Thus we must necessarily have $y^{\prime}<0$, and so, by (19), $x^{\prime}<0$ and hence the boundedness above of $x(\tau)$. This concludes the proof of Lemma 3 .

An immediate consequence of Lemmas 2 and 3 is the following

Corollary 1. Every solution to (14) with positive initial data is bounded and bounded away from zero.

Remark 1. The conclusions of Lemmas 2 and 3 (and, hence, also of Corollary 1) still hold true if the initial condition is non-negative, provided we consider, for the boundedness away from zero, times $\tau$ in $\left[\tau_{0}^{*}, \infty\right)$ for every $\tau_{0}^{*}>\tau_{0}$, where $\tau_{0}^{*}$ is the new initial time. This follows easily from the proof of Lemma 2 by taking $\left(\tau_{0}^{*}, x\left(\tau_{0}^{*} ; \tau_{0}, x_{0}, y_{0}\right), y\left(\tau_{0}^{*} ; \tau_{0}, x_{0}, y_{0}\right)\right)$ the new initial data, where now, from the proof of Lemma 1, both $x\left(\tau_{0}^{*}\right)$ and $y\left(\tau_{0}^{*}\right)$ are positive.

Having succeeded in proving every orbit of (14) is bounded and bounded away from zero, we can now start to identify its $\omega$-limit set. The first step towards this goal is the following lemma, which guarantees the $\omega$-limit set of every orbit is contained in the hyperbola $\{x y=1\}$. This will be achieved by considering the function

$$
h(\tau):=x(\tau) y(\tau),
$$

which is a very natural quantity to study if we keep in mind the role played by the hyperbola $\{x y=1\}$ in the proof of Lemma 3, as well as the form of Eq. (14).

Let $(x, y)$ be a solution of (14), then it is easy to conclude that $h(\tau)$ solves the equation

$$
h^{\prime}=\left(y+A \tau^{-\frac{1}{2}} x\right)-\left(y+2 A \tau^{-\frac{1}{2}} x+\left(A^{2}-B\right) \tau^{-1}\right) h .
$$

Observe that, rather surprisingly, this equation is linear in $h$. This allows the use of the variation of constants formula in order to gain precise knowledge of the behaviour of $h$. In fact, in the next lemma we not only prove that $h(\tau)$ converges to 1 as $\tau \rightarrow+\infty$, but that $h(\tau) \rightarrow$ $1^{-}$, i.e., for all sufficiently large $\tau$ the orbit remains in $\Omega_{+}$. Actually, our proof establishes a bit more: it gives the rate of convergence of $h(\tau)$ to 1 . Although this level of detail is not necessary at this point, we shall need it later on, in Lemma 7, in order to prove part (iii) of Theorem 1.

Lemma 4. Let $(x, y)$ be any solution to (14). Let $h$ be any solution to (21). Then $h(\tau) \rightarrow 1^{-}$as $\tau \rightarrow+\infty$. 
Proof. To simplify notation we introduce $\alpha(\tau):=y(\tau)+2 A \tau^{-\frac{1}{2}} x(\tau)+$ $\left(A^{2}-B\right) \tau^{-1}$. The variation of constants formula applied to (21) results in

$$
h(\tau)=h\left(\tau_{0}\right) e^{-\int_{\tau_{0}}^{\tau} \alpha(s) d s}+\int_{\tau_{0}}^{\tau}\left(y(s)+A s^{-\frac{1}{2}} x(s)\right) e^{-\int_{s}^{\tau} \alpha(\theta) d \theta} d s .
$$

We can write the second term in the right-hand side of this expression as follows

$$
\begin{aligned}
\int_{\tau_{0}}^{\tau}\left(y(s)+A s^{-\frac{1}{2}} x(s)\right) e^{-\int_{s}^{\tau} \alpha(\theta) d \theta} d s \\
=e^{-\int_{\tau_{0}}^{\tau} \alpha(s) d s} \int_{\tau_{0}}^{\tau} \frac{d}{d s}\left(e^{\int_{\tau_{0}}^{s} \alpha(\theta) d \theta}\right) d s \\
\quad-\int_{\tau_{0}}^{\tau}\left(\frac{A x(s)}{s^{1 / 2}}+\frac{A^{2}-B}{s}\right) e^{-\int_{s}^{\tau} \alpha(\theta) d \theta} d s \\
=1-e^{-\int_{\tau_{0}}^{\tau} \alpha(\theta) d \theta}-\int_{\tau_{0}}^{\tau}\left(\frac{A x(s)}{s^{1 / 2}}+\frac{A^{2}-B}{s}\right) e^{-\int_{s}^{\tau} \alpha(\theta) d \theta} d s
\end{aligned}
$$

and use this to rewrite (22) in the form

$$
\frac{1-h(\tau)}{A \tau^{-\frac{1}{2}}}=\frac{\left(1-h\left(\tau_{0}\right)\right)}{A \tau^{-\frac{1}{2}}} e^{-\int_{\tau_{0}}^{\tau} \alpha(s) d s}+\frac{\tau^{\frac{1}{2}}}{A} \int_{\tau_{0}}^{\tau}\left(\frac{A x(s)}{s^{1 / 2}}+\frac{A^{2}-B}{s}\right) e^{-\int_{s}^{\tau} \alpha(\theta) d \theta} d s
$$

It is important to observe that the first term in the right-hand-side of this expression is negative if the initial data for (14) is in $\Omega_{-}$. Also, by (15) and $(16)$, we have $\operatorname{sgn}\left(A^{2}-B\right)=\operatorname{sgn}(\omega)$ and so the second term in the integral can be a negative function. We shall prove that both these possibly negative contributions converge to zero as $\tau \rightarrow+\infty$, while the remaining one, which is always positive, is bounded and bounded away from zero.

By the boundedness away from zero of $y$ we can write $y(\tau) \geqslant L_{y}>0$ for some constant $L_{y}$, and get

$$
\begin{aligned}
e^{-\int_{\tau_{0}}^{\tau} \alpha(s) d s} & =\left(\frac{\tau_{0}}{\tau}\right)^{A^{2}-B} e^{-\int_{\tau_{0}}^{\tau}\left(y(s)+2 A s^{-1 / 2} x(s)\right) d s} \leqslant\left(\frac{\tau_{0}}{\tau}\right)^{A^{2}-B} e^{-\int_{\tau_{0}}^{\tau} y(s) d s} \\
& \leqslant\left(\frac{\tau_{0}}{\tau}\right)^{A^{2}-B} e^{-L_{y}\left(\tau-\tau_{0}\right)}
\end{aligned}
$$

Thus, as $\tau \rightarrow+\infty$, the first-term in the right-hand-side of (23) converges to zero. 
We next establish that

$$
\tau^{\frac{1}{2}} \int_{\tau_{0}}^{\tau} s^{-1} e^{-\int_{s}^{\tau} \alpha(\theta) d \theta} d s \rightarrow 0 \quad \text { as } \quad \tau \rightarrow+\infty
$$

Fix $\beta \in(0,1)$ and write the integral in (25) as $\int_{\tau_{0}}^{\tau-\tau^{\beta}}+\int_{\tau-\tau^{\beta}}^{\tau}$. The first inequality in (24) implies that the first of these integrals can be estimated as

$$
\begin{aligned}
& \tau^{\frac{1}{2}} \int_{\tau_{0}}^{\tau-\tau^{\beta}} s^{-1} e^{-\int_{s}^{\tau} \alpha(\theta) d \theta} d s< \\
& <\tau^{-\frac{1}{2}} \int_{\tau_{0}}^{\tau-\tau^{\beta}}\left(\frac{s}{\tau}\right)^{A^{2}-B-1} e^{-\int_{s}^{\tau} y(\theta) d \theta} d s \\
& <\tau^{-\frac{1}{2}} \max \left\{1,\left(\frac{\tau_{0}}{\tau}\right)^{A^{2}-B-1}\right\} \int_{\tau_{0}}^{\tau-\tau^{\beta}} e^{-L_{y}(\tau-s)} d s \\
& =\tau^{-\frac{1}{2}} \max \left\{1,\left(\frac{\tau_{0}}{\tau}\right)^{A^{2}-B-1}\right\} \frac{1}{L_{y}}\left(e^{-L_{y} \tau^{\beta}}-e^{-L_{y}\left(\tau-\tau_{0}\right)}\right) .
\end{aligned}
$$

For the second of the integrals above we have, again by (24),

$$
\begin{aligned}
& \tau^{\frac{1}{2}} \int_{\tau-\tau^{\beta}}^{\tau} s^{-1} e^{-\int_{s}^{\tau} \alpha(\theta) d \theta} d s< \\
& <\tau^{-\frac{1}{2}} \int_{\tau-\tau^{\beta}}^{\tau}\left(\frac{s}{\tau}\right)^{A^{2}-B-1} e^{-\int_{s}^{\tau} y(\theta) d \theta} d s \\
& <\tau^{-\frac{1}{2}} \max \left\{1,\left(1-\tau^{\beta-1}\right)^{A^{2}-B-1}\right\} \int_{\tau-\tau^{\beta}}^{\tau} e^{-L_{y}(\tau-s)} d s \\
& =\tau^{-\frac{1}{2}} \max \left\{1,\left(1-\tau^{\beta-1}\right)^{A^{2}-B-1}\right\} \frac{1}{L_{y}}\left(1-e^{-L_{y} \tau^{\beta}}\right) .
\end{aligned}
$$

Clearly, both (26) and (27) converge to zero as $\tau \rightarrow+\infty$, thus proving (25). To complete the proof we just need to conclude that there exists constants $L$ and $U$ such that, for all $\tau$ sufficiently large,

$$
0<L \leqslant \tau^{\frac{1}{2}} \int_{\tau_{0}}^{\tau} \frac{x(s)}{s^{\frac{1}{2}}} e^{-\int_{s}^{\tau} \alpha(\theta) d \theta} d s \leqslant U<+\infty
$$


Using the definition of $\alpha(\cdot)$ and the equation for $x$ in (14) we have

$$
\begin{aligned}
\alpha(\theta) & =\frac{1-x^{\prime}}{x}+\frac{A x}{\theta^{\frac{1}{2}}}+\frac{A^{2}}{\theta} \\
& =-\frac{d}{d \theta} \log x(\theta)+\frac{1}{x}+\frac{A x}{\theta^{\frac{1}{2}}}+\frac{A^{2}}{\theta},
\end{aligned}
$$

whence

$$
e^{-\int_{s}^{\tau} \alpha(\theta) d \theta}=\frac{x(\tau)}{x(s)}\left(\frac{s}{\tau}\right)^{A^{2}} \exp \left[-\int_{s}^{\tau}\left(\frac{1}{x(\theta)}+\frac{A x(\theta)}{\theta^{1 / 2}}\right) d \theta\right] .
$$

Plugging this into the integral in (28), and taking into account the fact that, by Corollary 1 , there exists constants $0<L_{x} \leqslant U_{x}$ such that $L_{x} \leqslant$ $x(\tau) \leqslant U_{x}$, we conclude that

$$
L_{x} J(\tau) \leqslant \tau^{\frac{1}{2}} \int_{\tau_{0}}^{\tau} \frac{x(s)}{s^{\frac{1}{2}}} e^{-\int_{s}^{\tau} \alpha(\theta) d \theta} d s \leqslant U_{x} J(\tau),
$$

where

$$
J(\tau):=\int_{\tau_{0}}^{\tau}\left(\frac{s}{\tau}\right)^{A^{2}-\frac{1}{2}} e^{-\int_{s}^{\tau}\left(\frac{1}{x(\theta)}+\frac{A x(\theta)}{\theta^{1 / 2}}\right) d \theta} d s
$$

and so it is sufficient to establish the bound (28) for $J(\tau)$.

As in the proof of $(25)$ we start by fixing $\beta \in(0,1)$ and writing the integral as a sum of a "small" and a "large" $\tau$ contribution, with $\tau-\tau^{\beta}$ as the threshold size. For the "small" size contribution we have, analogously to $(26)$,

$$
\begin{aligned}
& \int_{\tau_{0}}^{\tau-\tau^{\beta}}\left(\frac{s}{\tau}\right)^{A^{2}-\frac{1}{2}} e^{-\int_{s}^{\tau}\left(\frac{1}{x(\theta)}+\frac{A x(\theta)}{\theta^{1} / 2}\right) d \theta} d s \\
& <\int_{\tau_{0}}^{\tau-\tau^{\beta}}\left(\frac{s}{\tau}\right)^{A^{2}-\frac{1}{2}} e^{-\ell_{x}(\tau-s)} d s \\
& <\max \left\{1,\left(\frac{\tau_{0}}{\tau}\right)^{A^{2}-\frac{1}{2}}\right\} \frac{1}{\ell_{x}}\left(e^{-\ell_{x} \tau^{\beta}}-e^{-\ell_{x}\left(\tau-\tau_{0}\right)}\right),
\end{aligned}
$$

where $\ell_{x}:=\inf _{\theta \in\left[\tau_{0}, \infty\right)}\left(\frac{1}{x(\theta)}+\frac{A x(\theta)}{\theta^{1 / 2}}\right)$ is a positive constant, by Corollary 1 (and Remark 1). Clearly (29) converges to zero as $\tau \rightarrow+\infty$. For the "large" $\tau$ contribution, observe that, for $s \in\left[\tau-\tau^{\beta}, \tau\right]$,

$$
\min \left\{1,\left(1-\tau^{\beta-1}\right)^{A^{2}-\frac{1}{2}}\right\} \leqslant\left(\frac{s}{\tau}\right)^{A^{2}-\frac{1}{2}} \leqslant \max \left\{1,\left(1-\tau^{\beta-1}\right)^{A^{2}-\frac{1}{2}}\right\}
$$


and, since both the lower and the upper bounds converge to 1 as $\tau \rightarrow$ $+\infty$, to complete the proof it is sufficient to conclude that (28) is valid for the integral

$$
\int_{\tau-\tau^{\beta}}^{\tau} e^{-\int_{s}^{\tau}\left(\frac{1}{x(\theta)}+\frac{A x(\theta)}{\theta^{1 / 2}}\right) d \theta} d s
$$

With $\ell_{x}>0$ defined above, and $u_{x}:=\sup _{\theta \in\left[\tau_{0}, \infty\right)}\left(\frac{1}{x(\theta)}+\frac{A x(\theta)}{\theta^{1 / 2}}\right)<+\infty$, we immediately conclude that

$$
\frac{1-e^{-u_{x} \tau_{0}^{\beta}}}{u_{x}}<\frac{1-e^{-u_{x} \tau^{\beta}}}{u_{x}} \leqslant \int_{\tau-\tau^{\beta}}^{\tau} e^{-\int_{s}^{\tau}\left(\frac{1}{x(\theta)}+\frac{A x(\theta)}{\theta^{1 / 2}}\right) d \theta} d s \leqslant \frac{1-e^{-\ell_{x} \tau^{\beta}}}{\ell_{x}}<\frac{1}{\ell_{x}},
$$

which concludes the proof.

Having identified the $\omega$-limit set of every orbit as a subset of the hyperbola $\{x y=1\}$ and knowing, by Corollary 1 , that this set must be a bounded one, the next result provides a more precise knowledge of its location by showing it must be an (eventually degenerate) arc of the hyperbola containing the point $(1,1)$. This is achieved by the study of the dynamics of another auxiliary function, namely

$$
b(\tau):=y(\tau)-A \tau^{-\frac{1}{2}} x(\tau) .
$$

This function is a somewhat less natural choice than $h(\tau)$ used previously. The rationale for its definition is, nevertheless, not difficult to understand: the sets $\{h=$ const $\}$ provide a foliation of $\left(\mathbb{R}^{+}\right)^{2}$ and, since Lemma 4 shows the time behaviour of $h$ to be rather tame, it is reasonable to look for another variable, call it $b=b(\tau)$, so that every point $(x, y)$ is uniquely described by the new coordinates $(h, b)$. One possible definition, among others, is to have $b$ as the ordinate of the straight line $y=x+b$. However, due to the form of (14), the equation for this $b$ becomes rather unyielding. A better choice, from the point of view of handling the dynamic equation for the new variable, is the slight modification of this idea provided by (31).

It is easy to check that if $(x, y)$ is a solution of $(14)$ then $b(\tau)$ defined above solves the equation

$$
b^{\prime}=\left(-b+x^{2}\right) A^{2} \tau^{-1} .
$$

Changing the time scale $\tau \mapsto \zeta$ with $\frac{d \zeta}{d \tau}=A^{2} \tau^{-1}$, and still denoting by $b$ and $x$ those functions in the new independent variable, the equation for $b$ becomes

$$
\frac{d b}{d \zeta}=-b+x^{2}
$$


Again, the fact that this evolution equation is linear, and furthermore that it does not depend on $y$, allow us to use it to gain the sought for information about the location of the $\omega$-limit set.

Lemma 5. Let $(x, y)$ be any solution to (14). Let $x_{*}:=\varliminf_{\tau \rightarrow+\infty} x(\tau)$, $x^{*}:=\varlimsup_{\tau \rightarrow+\infty} x(\tau)$, and similarly for $y_{*}$ and $y^{*}$. Then, the following inequalities are satisfied

$$
x_{*} \leqslant 1 \leqslant x^{*} \quad \text { and } \quad y_{*} \leqslant 1 \leqslant y^{*} .
$$

Proof. Let $b$ be the function defined in (31). Fix any $\beta \in(0,1)$ and integrate (33) in $\left[\zeta-\zeta^{\beta}, \zeta\right]$ to obtain

$$
b(\zeta)=b\left(\zeta-\zeta^{\beta}\right) e^{-\zeta^{\beta}}+\int_{\zeta-\zeta^{\beta}}^{\zeta} x^{2}(s) e^{-(\zeta-s)} d s .
$$

The first-term in the right-hand side of (34) converges to zero as $\zeta \rightarrow$ $+\infty$ because the exponential converges to zero, and (31) together with the boundedness of $x$ and $y$ entail the boundedness of $b\left(\zeta-\zeta^{\beta}\right)$. The integral in (34) can be bounded above using the supremum of $x^{2}$ in the region of integration to obtain, as $\zeta \rightarrow+\infty$,

$$
\begin{aligned}
b(\zeta) & \leqslant \mathcal{O}(1)+\left(\sup _{s \in\left[\zeta-\zeta^{\beta}, \zeta\right]} x^{2}(s)\right) \int_{\zeta-\zeta^{\beta}}^{\zeta} e^{-(\zeta-s)} d s \\
& \leqslant \mathcal{O}(1)+\left(1-e^{-\zeta^{\beta}}\right) \sup _{s \in\left[\zeta-\zeta^{\beta}, \infty\right)} x^{2}(s),
\end{aligned}
$$

and taking $\overline{\lim }_{\zeta \rightarrow+\infty}$ we obtain

$$
\varlimsup_{\zeta \rightarrow+\infty} b(\zeta) \leqslant\left(x^{*}\right)^{2} .
$$

Now, taking again (34), using this time the bound with the infimum of $x^{2}$ in the region of integration and taking $\underline{\lim }_{\zeta \rightarrow+\infty}$ we get the corresponding inequality

$$
\underline{\lim }_{\zeta \rightarrow+\infty} b(\zeta) \geqslant\left(x_{*}\right)^{2} .
$$

From the definition of $b$ and the boundedness of $x$ we also know that

$$
\varlimsup_{\zeta \rightarrow+\infty} b(\zeta)=y^{*}, \quad \underline{\lim }_{\zeta \rightarrow+\infty} b(\zeta)=y_{*} .
$$




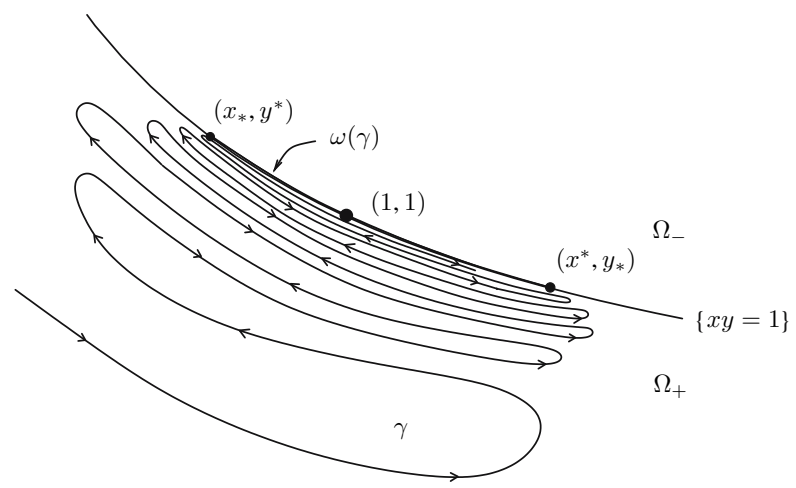

Figure 4. Long time behaviour of the orbits if $x_{*}<1<x^{*}$.

Furthermore, since by Lemma 4 we know that $h(\zeta) \rightarrow 1$ as $\zeta \rightarrow+\infty$, we also have

$$
y^{*}=\frac{1}{x_{*}} \quad \text { and } \quad y_{*}=\frac{1}{x^{*}} .
$$

Now, using (35), (37) (38), and $x_{*} \leqslant x^{*}$, we conclude that

$$
\frac{1}{x^{*}} \leqslant \frac{1}{x_{*}}=y^{*}=\varlimsup_{\zeta \rightarrow+\infty} b(\zeta) \leqslant\left(x^{*}\right)^{2}
$$

and so $x^{*} \geqslant 1$. Similarly, we have the corresponding result using (36), namely $x_{*} \leqslant 1$. From these inequalities and (38) we conclude the proof.

We are now left with the need to prove the equalities in the conclusion of Lemma $5 \mathrm{do}$, in fact, hold. This will be done next

Lemma 6. Let $(x, y)$ be any solution to (14). Then, with the notation of Lemma 5 ,

$$
x_{*}=1=x^{*} \quad \text { and } \quad y_{*}=1=y^{*} .
$$

Proof. We shall assume $x_{*}<1<x^{*}$ and draw a contradiction. First notice that in this situation every orbit $\gamma$ will eventually behave like the one depicted in Fig. 4.

Pick any $(\tilde{x}, \tilde{y})$ in the hyperbola $\{x y=1\}$ located strictly between $(1,1)$ and $\left(x_{*}, y^{*}\right)$. Since $(\widetilde{x}, \widetilde{y}) \in \omega(\gamma)$, and from the geometry of the situation, we can choose a sequence $\tau_{n} \uparrow+\infty$ and a corresponding sequence of points $P_{n}=\left(x\left(\tau_{n}\right), y\left(\tau_{n}\right)\right) \in \gamma$ satisfying $P_{n} \underset{n \rightarrow+\infty}{\longrightarrow}(\tilde{x}, \tilde{y})$, and $x^{\prime}\left(\tau_{n}\right)<0$ and 


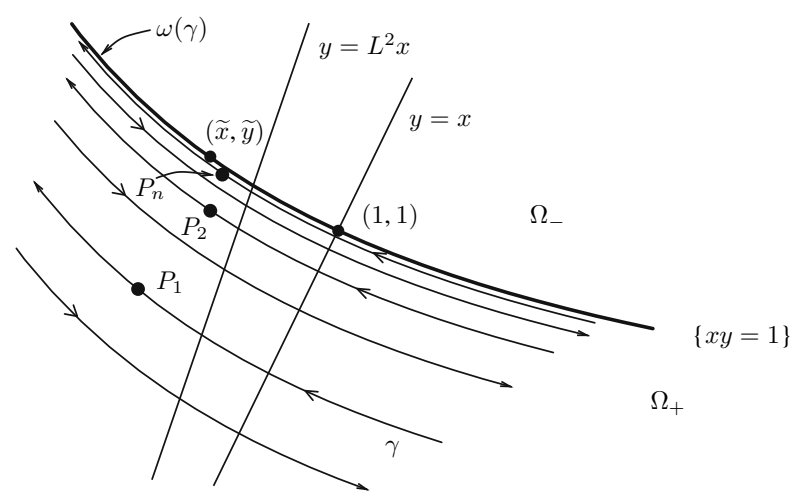

Figure 5. Sequence of points $\left(P_{n}\right)$ on $\gamma$ converging to $(\tilde{x}, \tilde{y}) \in \omega(\gamma)$.

$y^{\prime}\left(\tau_{n}\right)>0$ for all $n$. Furthermore, since $x\left(\tau_{n}\right) \rightarrow \tilde{x}<1$ and $y\left(\tau_{n}\right) \rightarrow \tilde{y}>1$, we can, without loss of generality, consider a sequence satisfying $\frac{y\left(\tau_{n}\right)}{x\left(\tau_{n}\right)}>L^{2}>1$ for all $n$, and for some constant $L$ independent of $n$ (see Fig. 5).

We easily conclude that inequality (18) holds true for the coordinates of the points $P_{n}$, namely

$$
y^{\prime}\left(\tau_{n}\right)<x^{\prime}\left(\tau_{n}\right) A \tau_{n}^{-\frac{1}{2}}
$$

But then $x^{\prime}\left(\tau_{n}\right)<0 \Rightarrow y^{\prime}\left(\tau_{n}\right)<0$ contradicting our assumption about the $P_{n}$. This contradiction shows there is no sequence satisfying those conditions, which can only be so if we cannot choose $(\widetilde{x}, \widetilde{y})$ as stated, i.e., if $\left(x_{*}, y^{*}\right)=(1,1)$.

Now choose any point $(\widehat{x}, \widehat{y})$ in $\{x y=1\}$ strictly between $(1,1)$ and $\left(x^{*}, y_{*}\right)$. Again we can choose a sequence of times $v_{n} \uparrow+\infty$ and a corresponding sequence $Q_{n}=\left(x\left(v_{n}\right), y\left(v_{n}\right)\right) \in \gamma$ satisfying $Q_{n} \underset{n \rightarrow+\infty}{\longrightarrow}(\widehat{x}, \widehat{y})$, but this time such that $x^{\prime}\left(v_{n}\right)>0$ and $y^{\prime}\left(v_{n}\right)<0$ for all $n$. Without loss of generality we consider $x\left(v_{n}\right)>1$ for all $n$ (see Fig. 6).

To proceed we need to obtain an inequality relating $x^{\prime}\left(v_{n}\right)$ and $y^{\prime}\left(v_{n}\right)$ from which to obtain a contradiction. From (14) and $x\left(v_{n}\right)>1 \Rightarrow x\left(v_{n}\right)^{2}>$ $x\left(v_{n}\right)$ we conclude that, for all $n$ sufficiently large

$$
\begin{aligned}
y^{\prime} & =\left(1-x y-A v_{n}^{-\frac{1}{2}} y\right) A v_{n}^{-\frac{1}{2}} \\
& =\left(x^{\prime}+A v_{n}^{-\frac{1}{2}} x^{2}-B v_{n}^{-1} x-A v_{n}^{-\frac{1}{2}} y\right) A v_{n}^{-\frac{1}{2}}
\end{aligned}
$$




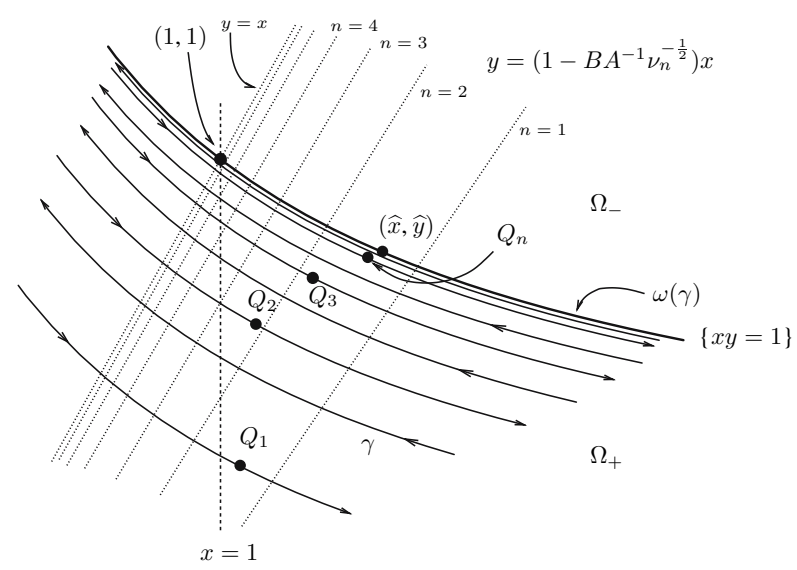

Figure 6. Sequence of points $\left(Q_{n}\right)$ on $\gamma$ converging to $(\widehat{x}, \widehat{y}) \in \omega(\gamma)$.

$$
\begin{aligned}
& >\left(x^{\prime}+\left(A v_{n}^{-\frac{1}{2}}-B v_{n}^{-1}\right) x-A v_{n}^{-\frac{1}{2}} y\right) A v_{n}^{-\frac{1}{2}} \\
& =\left(x^{\prime}+\left[\left(1-B A^{-1} v_{n}^{-\frac{1}{2}}\right) x-y\right] A v_{n}^{-\frac{1}{2}}\right) A v_{n}^{-\frac{1}{2}} \\
& >x^{\prime} A v_{n}^{-\frac{1}{2}}
\end{aligned}
$$

where we wrote $x$ for $x\left(v_{n}\right)$ and $y$ for $y\left(v_{n}\right)$ in order not to overload the notation. For the last inequality two observations are in order: if $\omega \geqslant 1$ then $B \leqslant 0$ and thus, for all $n$, the straight lines $y=\left(1-B A^{-1} v_{n}^{-\frac{1}{2}}\right) x$ are above $y=x$, and so $y\left(v_{n}\right)<\left(1-B A^{-1} v_{n}^{-\frac{1}{2}}\right) x\left(v_{n}\right)$, which imply the last inequality in (39); if $\omega \in\left(-\frac{1}{2}, 1\right)$ then $B>0$, in which case the straight lines $y=\left(1-B A^{-1} v_{n}^{-\frac{1}{2}}\right) x$, although below $y=x$, converge to this line uniformly in compact sets, and thus, for all sufficiently large values of $n$, we again have the inequality (39). Whence, we conclude from (39) and the assumption $x^{\prime}\left(v_{n}\right)>0$ that also $y^{\prime}\left(v_{n}\right)>0$, a contradiction that shows there is no sequence satisfying the conditions, which in turn imply no such $(\widehat{x}, \widehat{y})$ exists, i.e., that $\left(x^{*}, y_{*}\right)=(1,1)$. This concludes the proof.

At this point we have all the ingredients to actually complete the proof of parts (i) and (ii) of Theorem 1. Part (iii) requires a more precise 
knowledge of how $h(\tau)$ converges to 1 as $\tau \rightarrow+\infty$, and this will be the object of the next and final lemma of this section.

Lemma 7. Let $(x, y)$ be any solution to (14) and let $h=x y$. Then we have

$$
\lim _{\tau \rightarrow+\infty} \frac{1-h(\tau)}{A \tau^{-\frac{1}{2}}}=1
$$

Proof. The proof is equal to that of Lemma 4 down to the estimate (28), which now must be changed to

$$
\tau^{\frac{1}{2}} \int_{\tau_{0}}^{\tau} \frac{x(s)}{s^{\frac{1}{2}}} e^{-\int_{s}^{\tau} \alpha(\theta) d \theta} d s \longrightarrow 1 \quad \text { as } \quad \tau \rightarrow+\infty
$$

the analysis of which also follows the same lines as in the proof of Lemma 4 , but now we make use of $x(\tau)=1+\mathcal{O}(1)$ to relate the integral in (40) with $J(\tau)$. In the end, corresponding to the estimate of (30), we now need to prove that

$$
\int_{\tau-\tau^{\beta}}^{\tau} e^{-\int_{s}^{\tau}\left(\frac{1}{x(\theta)}+\frac{A x(\theta)}{\theta^{1 / 2}}\right) d \theta} d s \longrightarrow 1 \quad \text { as } \quad \tau \rightarrow+\infty
$$

and for this we shall use the convergence of $x(\tau)$ to 1 provided by Lemma 6. From this convergence we can write that

$$
\forall_{\varepsilon>0}, \quad \exists_{T^{*}}: \forall_{\theta}, \quad \theta>T^{*} \Rightarrow 1-\frac{\varepsilon}{2}<\frac{1}{x(\theta)}<1+\frac{\varepsilon}{2}
$$

and also that

$$
\forall_{\varepsilon>0}, \exists_{T^{* *}}: \forall_{\theta}, \theta>T^{* *} \Rightarrow-\frac{\varepsilon}{2}<\frac{A x(\theta)}{\theta^{\frac{1}{2}}}<\frac{\varepsilon}{2} .
$$

Consequently, for all $\theta>T:=\max \left\{T^{*}, T^{* *}\right\}$, we have

$$
1-\varepsilon<\frac{1}{x(\theta)}+\frac{A x(\theta)}{\theta^{\frac{1}{2}}}<1+\varepsilon,
$$

which implies that, by considering $\tau$ so large that $\tau-\tau^{\beta}>T$, we have $\theta \geqslant$ $s \geqslant \tau-\tau^{\beta}>T$ and so the integral in (41) can be estimated as

$$
\frac{1}{1+\varepsilon}\left(1-e^{-(1+\varepsilon) \tau^{\beta}}\right) \leqslant \int_{\tau-\tau^{\beta}}^{\tau} e^{-\int_{s}^{\tau}\left(\frac{1}{x(\theta)}+\frac{A x(\theta)}{\theta^{1 / 2}}\right) d \theta} d s \leqslant \frac{1}{1-\varepsilon}\left(1-e^{-(1-\varepsilon) \tau^{\beta}}\right) .
$$

Passing to the limit $\tau \rightarrow+\infty$, and by the arbitrariness of $\varepsilon>0$, we immediately conclude (41). 
We are now in position to prove Theorem 1:

Proof of Theorem 1. Lemma 6 means that $(x(\tau), y(\tau)) \rightarrow(1,1)$ as $\tau \rightarrow$ $+\infty$ and thus, by (10)-(13), we conclude statements (i) and (ii) of the theorem hold true. To prove (iii), first remember the relation between the two time scales: $\frac{d \tau}{d t}=\left(\frac{3 \alpha^{2}}{1+2 \omega}\right)^{\frac{1}{3}} t^{\frac{1+2 \omega}{3}}$. After integration this obviously entails, as $\tau \rightarrow+\infty$ (or as $t \rightarrow+\infty$ ),

$$
\tau^{-\frac{1}{2}} t^{\frac{2+\omega}{3}}\left(\frac{3 \alpha^{2}}{1+2 \omega}\right)^{\frac{1}{6}}\left(\frac{3}{4+2 \omega}\right)^{\frac{1}{2}}=1+\mathcal{O}(1)
$$

Now, using (10)-(13), (15) and (42), we get, after a few algebraic manipulations,

$$
\frac{1-h(\tau)}{A \tau^{-\frac{1}{2}}}=\left(\frac{3}{\alpha(1+2 \omega)}\right)^{\frac{2}{3}} t^{2 \frac{1-\omega}{3}}\left(\alpha t^{\omega}-c_{0}(t) c_{1}(t)\right)(1+\mathcal{O}(1)) \quad \text { as } \tau, t \rightarrow+\infty .
$$

By Lemma 7 the left-hand side of this expression is $1+\mathcal{O}(1)$ and this completes the proof.

\section{LONG TIME BEHAVIOUR}

From now on we consider the time scale introduced in (7). The first result we need is the following auxiliary statement concerning the relation between the original $t$-scale and the new $\varsigma$-scale defined by (7).

In order to simplify the notation, we first define the following time independent scaling factor

$$
Q(\omega):=\left(\frac{3}{\alpha(1+2 \omega)}\right)^{\frac{1}{2+\omega}}\left(\frac{2+\omega}{3}\right)^{r}, \quad \text { where } r:=\frac{1-\omega}{2+\omega} .
$$

Proposition 1. With $\left(c_{j}\right), \varsigma$, and $\left(\widetilde{c}_{j}\right)$ given in (7) and (8) and $Q(\omega)$ given by (43), the following holds true:

$$
\begin{array}{ll}
\text { (i) } & \lim _{t \rightarrow+\infty} \frac{2+\omega}{3}\left(\frac{3}{\alpha(1+2 \omega)}\right)^{1 / 3} t^{-\frac{2+\omega}{3}} \varsigma(t)=1, \\
\text { (ii) } & \lim _{\varsigma \rightarrow+\infty} Q(\omega) \varsigma^{r} \widetilde{c}_{1}(\varsigma)=1 .
\end{array}
$$

Sketch of the Proof. The proof is essentially that of da Costa et al. [5, Proposition 3] and so we shall present only the main idea. From 
Theorem 1 (ii) we have the following bounds

$$
\forall \varepsilon>0, \quad \exists T=T(\varepsilon): \forall t>T(\varepsilon), \quad 1-\varepsilon<\left(\frac{3}{\alpha(1+2 \omega)}\right)^{\frac{1}{3}} t^{\frac{1-\omega}{3}} c_{1}(t)<1+\varepsilon .
$$

First consider the upper bound, substitute it into (7), integrate between $t$ and $t_{0}$ and apply $\overline{\lim }_{t \rightarrow+\infty}$. Then repeat the process taking the lower bound and applying $\underline{\lim }_{t \rightarrow+\infty}$. The two results thus obtained, together with the arbitrariness of $\varepsilon$, prove part (i). To get part (ii), again start from Theorem 1 (ii), and use part (i) above to write, as $t, \varsigma \rightarrow+\infty$,

$$
\begin{aligned}
1+\mathcal{O}(1) & =\left(\frac{3}{\alpha(1+2 \omega)}\right)^{\frac{1}{3}} t^{\frac{1-\omega}{3}} c_{1}(t) \\
& =\left(\frac{3}{\alpha(1+2 \omega)}\right)^{\frac{1}{3}}\left[\left(\frac{2+\omega}{3}\left(\frac{3}{\alpha(1+2 \omega)}\right)^{\frac{1}{3}} \varsigma\right)^{\frac{3}{2+\omega}}\right]^{\frac{1-\omega}{3}} \widetilde{c}_{1}(\varsigma) \\
& =Q(\omega) \varsigma^{r} \widetilde{c}_{1}(\varsigma),
\end{aligned}
$$

which concludes the proof.

We can now use the expression (9) for the $\widetilde{c}_{j}(\varsigma)$ component of the solution, and the information provided by Proposition 1 to prove the following result about the asymptotic behaviour of solutions to (2):

Theorem 2. Let $\left(c_{j}\right)$ be any non-negative solution of (2) with initial data satisfying $c_{0}(0)=\sum_{j=1}^{\infty} c_{j}(0)<\infty$. Then, as $t \rightarrow+\infty$, we have

(i) $\left(\frac{3}{\alpha(1+2 \omega)}\right)^{\frac{1}{3}} t^{\frac{1-\omega}{3}} c_{j}(t) \longrightarrow 1 \quad$ for all $j \geqslant 1$,

$$
\begin{aligned}
& \left(\frac{1+2 \omega}{3 \alpha^{2}}\right)^{\frac{1}{3}} t^{-\frac{1+2 \omega}{3}} \sum_{j=1}^{\infty} c_{j}(t) \longrightarrow 1 \\
& \left(\frac{3}{\alpha(1+2 \omega)}\right)^{\frac{2}{3}} t^{2 \frac{1-\omega}{3}}\left(\alpha t^{\omega}-c_{1}(t) \sum_{j=1}^{\infty} c_{j}(t)\right) \longrightarrow 1 .
\end{aligned}
$$

Sketch of the Proof. Again in this case, the proof closely follows the one in [5], Section 4, and so we shall only present the main idea. For $j=1$, (i) is just (ii) from Theorem 1 . For $j \geqslant 2$ we can use the expression (9) for the $\widetilde{c}_{j}$ : multiplying both sides of (9) by $Q(\omega) \varsigma^{r}$ we have two terms; the first one can be dealt with exactly as in the $\omega=0$ case [5], Section 4 , since $Q(\omega) \varsigma^{r} e^{-\varsigma} \sum_{k=2}^{j} \frac{\varsigma^{j-k}}{(j-k) !} c_{k}(0)=\mathcal{O}\left(\varsigma^{j-1} e^{-\varsigma}\right)=\mathcal{O}\left(e^{-\lambda \varsigma}\right)$, for every $\lambda<1$. For the second one, a change of variables allow us to write the 
integral term as an integral over $[0,1]$ and then split it into two integrals, one over $[0,1-\varepsilon]$ and another over $[1-\varepsilon, 1]$, for a fixed $0<\varepsilon<1$. Define $\psi_{\omega}(\cdot)=Q(\omega)(\cdot)^{r} \widetilde{c}_{1}(\cdot)$, which is a continuous function, and $1+\mathcal{O}(1)$ at infinity by ( $i i)$ in Proposition 1 , and hence $\exists M_{\psi_{\omega}}: 0 \leqslant \psi_{\omega}(s) \leqslant M_{\psi_{\omega}}$ and we have

$$
\begin{aligned}
& \varsigma^{j-1} \int_{1-\varepsilon}^{1} \frac{\psi_{\omega}(y) y^{j-2}}{(1-y)^{r}} e^{-\varsigma y} d y \leqslant \\
& \leqslant M_{\psi_{\omega}} \varsigma^{j-1} e^{-(1-\varepsilon) \varsigma} \int_{1-\varepsilon}^{1} \frac{y^{j-2}}{(1-y)^{r}} d y<\frac{1}{1-r} \varepsilon^{1-r} M_{\psi_{\omega}} \varsigma^{j-1} e^{-(1-\varepsilon) \varsigma}
\end{aligned}
$$

and so it is also exponentially small as $\varsigma \rightarrow+\infty$. To understand the behaviour of the integral over $[0,1-\varepsilon]$ we make use of Watson's Lemma, just as in the $\omega=0$ case, to obtain

$$
\int_{0}^{1-\varepsilon} \frac{y^{j-2}}{(1-y)^{r}} e^{-\varsigma y} d y=\frac{\Gamma(j-1)}{\varsigma^{j-1}}+\mathcal{O}\left(\frac{1}{\varsigma^{j}}\right) \quad \text { as } \varsigma \rightarrow+\infty,
$$

since we can write $\frac{y^{j-2}}{(1-y)^{r}}=y^{j-2}\left(1+\sum_{j=1}^{\infty} \frac{y^{j}}{j !} \prod_{k=0}^{j-1}(r+k)\right)$, which is convergent for $|y|<1$. These three exponentially small terms allow us to conclude that $\lim _{\zeta \rightarrow+\infty} Q(\omega) \varsigma^{r} \widetilde{c}_{j}(\varsigma)=1$, for all $j \geqslant 2$, which is equivalent to (i) as is clear from the proof of Proposition 1 ( $i$ ).

Cases (ii) and ( $i$ i) are those already proved in Theorems 1 (i) and (iii), respectively.

\section{CONVERGENCE TO SELF-SIMILARITY}

We can now turn to the results concerning convergence of solutions to self-similar profiles. Let $\Phi_{1, \omega}: \mathbb{R}^{+} \backslash\{1\} \rightarrow \mathbb{R}$ be defined by

$$
\Phi_{1, \omega}(\eta):= \begin{cases}(1-\eta)^{\frac{\omega-1}{\omega+2}}, & \text { if } \eta<1 \\ 0, & \text { if } \eta>1\end{cases}
$$

In Fig. 7, we present the graphs of functions $\Phi_{1, \omega}$ for various values of $\omega$. Note these functions can be continuously extended to $\eta=1$ if and only if $\omega>1$.

Our first result states that the functions $\Phi_{1, \omega}$ are the similarity profiles of the solutions to (2) along non-characteristic directions.

Theorem 3. Let $\left(c_{j}\right)$ be any non-negative solution of (2) with initial data satisfying $\exists \rho>0, \mu>r: \forall j, c_{j}(0) \leqslant \rho / j^{\mu}$, where again $r:=\frac{1-\omega}{2+\omega}$. Let 


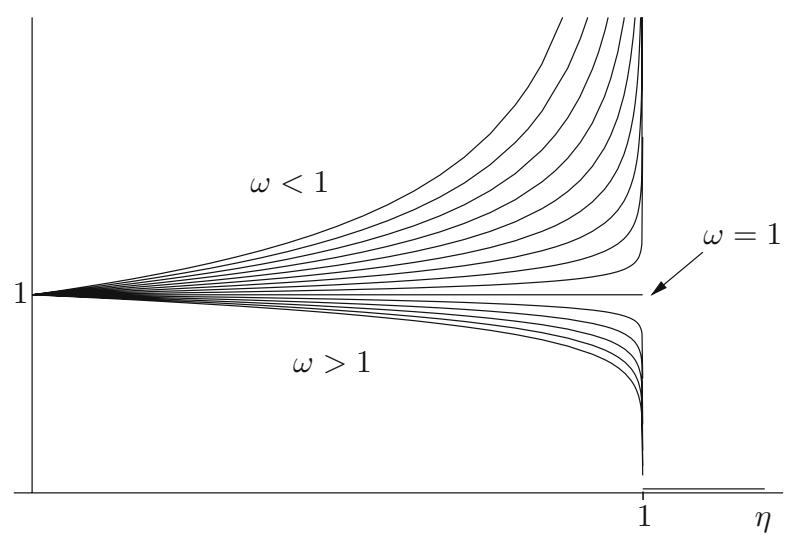

Figure 7. Graphs of $\Phi_{1, \omega}$ for $\omega=1$ and for values of $\omega$ below and above 1 in steps of 0.1

$\varsigma(t)$ and $\widetilde{c}_{j}(\varsigma)$ be as in (7) and (8), respectively, and let $Q(\omega)$ be given by (43). Then,

$$
\lim _{\substack{j, \zeta \rightarrow+\infty \\ \eta=j / \varsigma \text { fixed } \\ \eta \neq 1}} Q(\omega) \varsigma^{r} \widetilde{c}_{j}(\varsigma)=\Phi_{1, \omega}(\eta) .
$$

The proof of this theorem follows exactly the same steps as the proof of Theorem 3 in [5] where the corresponding result for the autonomous $\omega=0$ case was established. Later in this section we will describe those main steps and direct the reader to da Costa et al. [5], Section 5, for full details.

When $\omega \in\left(-\frac{1}{2}, 1\right]$ the similarity profiles $\Phi_{1, \omega}$ have a jump at $\eta=1$. In these cases it is natural to look for a different similarity variable and scaling that can provide a better description of the behaviour of solutions along the characteristic direction corresponding to the jump position $\eta=1$. As pointed out in the Introduction, one such similarity variable is $\xi \in \mathbb{R}$ defined by $j=\varsigma+\xi \sqrt{\varsigma}$, with $\varsigma$ given, as in Theorem 3, by expression (7). Let $\Phi_{2, \omega}: \mathbb{R} \rightarrow \mathbb{R}$ be defined by

$$
\Phi_{2, \omega}(\xi):=e^{-\frac{1}{2} \xi^{2}} \int_{0}^{+\infty} y^{\frac{3 \omega}{\omega+2}} e^{-\xi y^{2}-\frac{1}{2} y^{4}} d y .
$$

In Fig. 8 we present the graphs of $\Phi_{2, \omega}$ for several values of $\omega$ in $\left(-\frac{1}{2}, 1\right]$.

Our final result proves that the functions $\Phi_{2, \omega}$ are also similarity profiles of the solutions to (2). Observe that not only the similarity variable 


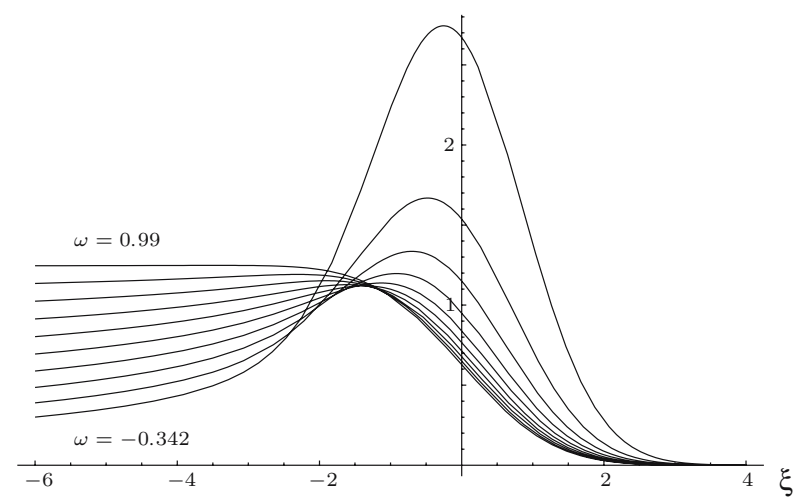

Figure 8. Graphs of $\Phi_{2, \omega}$ for $\omega$ from -0.342 to 0.99 in steps of 0.148 .

has changed, but the time scale is also different: the power of $\varsigma$ is half that of Theorem 3 .

Theorem 4. Let $\left(c_{j}\right)$ be any non-negative solution of (2) with monomeric initial data. Let $\varsigma(t), \widetilde{c}_{j}(\varsigma), Q(\omega)$, and $r$ be as in Theorem 3. Then,

$$
\lim _{\substack{j, \zeta \rightarrow+\infty \\ \xi=\frac{j-\varsigma}{\sqrt{\zeta}} \text { fixed } \\ \xi \in \mathbb{R}}}\left(\frac{\pi}{2}\right)^{\frac{1}{2}} Q(\omega) \varsigma^{\frac{r}{2}} \widetilde{c}_{j}(\varsigma)=\Phi_{2, \omega}(\xi) .
$$

Again, as in the proof of Theorem 3, the proof of this result is analogous to what was done in [5] with the corresponding result for the autonomous $\omega=0$ case. We shall only point out the main idea, full details can be checked in [5], Section 6.

Sketch of the Proof of Theorem 3. First consider the case of monomeric initial conditions $c_{j}(0)=c_{1}(0) \delta_{1, j}$. Multiplying (9) by $Q(\omega) \varsigma^{r}$, using Stirling's expansion, changing the integration variable linearly so that the integration region becomes $(0,1)$, and finally using Proposition 1 (ii), we obtain

$$
\begin{aligned}
\varphi_{1, \omega}(\eta, \varsigma):= & \frac{1}{\sqrt{2 \pi}} \eta^{\frac{3}{2}-\eta \varsigma} \varsigma^{\frac{1}{2}}\left(1+\mathcal{O}\left(\varsigma^{-1}\right)\right) \\
& \times \int_{0}^{1} \psi_{\omega}(\varsigma(1-y)) \frac{e^{\zeta(\eta \log y-y+\eta)}}{y^{2}(1-y)^{r}} d y,
\end{aligned}
$$

where $\psi_{\omega}(\cdot):=Q(\omega)(\cdot)^{r} \widetilde{c}_{1}(\cdot)$. To prove that $\varphi_{1, \omega}$ converges to $\Phi_{1, \omega}$ when $\varsigma \rightarrow+\infty$ (for fixed $\eta$ ) we proceed exactly as in [5], Section 5.1; the case 
$\eta>1$ is easy, since the integral in (46) can be estimated using the bound $y^{-2} e^{5(\eta \log y-y+\eta)}<e^{-\varsigma}$ and the $L^{1}(0,1)$-integrability of $\theta \mapsto \theta^{-r}$ arising from the fact that $r<1$ for $\omega>-\frac{1}{2}$. When $\eta \in(0,1)$ we decompose the integral in (46) as $\int_{0}^{\varepsilon}+\int_{\varepsilon}^{1-\varepsilon}+\int_{1-\varepsilon}^{1}$ with $\varepsilon<\min \left\{\eta e^{-1}, 1-\eta\right\}$. The contributions of the first and last integrals can be estimated as in the case $\eta>1$. In order to conclude the result we then need to prove that, as $\varsigma \rightarrow+\infty$, the integral on $(\varepsilon, 1-\varepsilon)$ converges to $\Phi_{1, \omega}(\eta)$. This follows from Proposition 1 (ii), which implies that $\psi_{\omega}(\theta)=1+\mathcal{O}(1)$ as $\theta \rightarrow+\infty$, when $\omega$ is fixed, and from Laplace's method for the asymptotic evaluation of integrals [1], p. 431. As pointed out above, the details should be looked up in [5], Section 5.1.

If the initial condition is not monomeric we have the contribution arising from the sum term in the right-hand-side of (9). Multiplying it by $Q(\omega) \varsigma^{r}$ we are now left to prove that

$$
\lim _{\substack{j, \zeta \rightarrow+\infty \\ \eta=j / \varsigma \text { fixed } \\ \eta \neq 1}} Q(\omega) \varsigma^{r} e^{-\varsigma} \sum_{k=2}^{j} \frac{\tau^{j-k}}{(j-k) !} c_{k}(0)=0
$$

Changing the summation variable $k \mapsto \ell:=j-k$, using the bound on the initial data, and using the similarity variable to write $\varsigma=j v$, with $v:=\eta^{-1}$, the limit (47) can be estimated by the limit when $j \rightarrow \infty$ of the function $\varphi_{2, \omega}(v, j):=(j v)^{r} e^{-j v} \sum_{\ell=0}^{j-2} \frac{(j v)^{\ell}}{\ell !(j-\ell)^{\mu}}$, the study of which proceeds exactly as was done in [5], Section 5.2, for the case $r=1 / 2$ (corresponding to $\omega=0$ ): now $r-\frac{1}{2}$ is not necessarily zero and we thus obtain the proof for the present case by multiplying all the estimates in [5], Section 5.2, by $(j v)^{r-\frac{1}{2}}$.

Sketch of the Proof of Theorem 4. Here, as in the case of Theorem 3, the proof follows step by step that presented in [5] for $\omega=0$. It proceeds by multiplying the integral in (9) by $\left(\frac{\pi}{2}\right)^{\frac{1}{2}} Q(\omega) 5^{\frac{r}{2}}$, changing the integration variable $s \mapsto w:=\sqrt{\sqrt{5}-\frac{s}{\sqrt{5}}}$, using Stirling's expansion and L'Hôpital's rule to write the multiplicative factor outside the integral as $\sim e^{-\frac{1}{2} \xi^{2}}$ when $\varsigma \rightarrow+\infty$, and, finally, by separating the integral into $\int_{0}^{\varepsilon}+\int_{\varepsilon}^{\varsigma^{1 / 4}}$ and estimating these integrals when $\varsigma \rightarrow+\infty$. The only difference from the results in [5], Section 6.1, is that now both integrals contain the multiplicative factor $y^{1-2 r}$ in the integrand function. As this factor does not depend on $\zeta$, the asymptotic estimates in [5] apply verbatim. 


\section{ACKNOWLEDGMENTS}

We would like to thank the anonymous referee for pointing out a loophope in the original proof of Lemma 3 and for suggesting a way to correct it.

Partially supported by Fundação para a Ciência e a Tecnologia through project PDCT/MAT/56476/2004.

\section{REFERENCES}

1. Ablowitz, M. J. and Fokas, A. S. (2003). Complex Variables, 2nd Edn., Cambridge Texts in Applied Mathematics. Cambridge University Press, Cambridge.

2. Ball, J. M., Carr, J. and Penrose, O. (1986). The Becker-Döring cluster equations: Basic properties and asymptotic behaviour of solutions. Comm. Math. Phys. 104, 657-692.

3. Bartelt, M. C. and Evans, J. W. (1996). Exact island-size distributions for submonolayer deposition: influence of correlations between island size and separation. Phys. Rev. B 54, R17359-R17362.

4. Brilliantov, N. V. and Krapivsky, P. L. (1991). Non-scaling and source-induced scaling behaviour in aggregation models of movable monomers and immovable clusters. J. Phys. A; Math. Gen. 24, 4787-4803.

5. da Costa, F.P., van Roessel, H., and Wattis, J. A. D., (2006). Long-time behaviour and self-similarity in a coagulation equation with input of monomers. Markov Processes Relat. Fields 12, 367-398.

6. Fournier, N., and Laurençot, Ph., (2005). Existence of self-similar solutions to Smoluchowski's coagulation equation. Commun. Math. Phys. 256, 589-609.

7. Hendriks, E. M., and Ernst, M. H., (1984). Exactly soluble addition and condensation models in coagulation kinetics. J. Coll. Int. Sci. 97, 176-194.

8. Laurençot, Ph., and Mischler, S. and (2004). On Coalescence equations and related models. In Degond, P., Pareschi, L., and Russo, G. (eds.), Modelling and Computational Methods for Kinetic Equations, Birkhäuser, Boston, pp. 321-356.

9. Leyvraz, F. (2003). Scaling theory and exactly solved models in the kinetics of irreversible aggregation, Phys. Rep. 383, 95-212.

10. Leyvraz, F. (2005). Rigorous results in the scaling theory of irreversible aggregation kinetics. J. Nonlinear Math. Phys. 12 (Suppl 1), 449-465.

11. Menon, G., and Pego, R. L., (2004). Approach to self-similarity in Smoluchowski's coagulation equations. Commun. Pure Appl. Math. 57, 1197-1232.

12. Menon, G., Pego, R. L. (2005). Dynamical scaling in Smoluchowski's coagulation equations: uniform convergence. SIAM J. Math. Anal. 36, 1629-1651.

13. Penrose, O. (1989). Metastable states for the Becker-Döring cluster equations. Commun. Math. Phys. 124, 515-541.

14. Wattis, J. A. D. (2004). Similarity solutions of a Becker-Döring system with time-dependent monomer input. J. Phys. A: Math. Gen. 37, 7823-7841. 